\title{
Steganalysis of JPEG Images with Joint Transform Features
}

\author{
Zohaib Khan and Atif Bin Mansoor \\ College of Aeronautical Engineering, \\ National University of Sciences \& Technology, Pakistan \\ zohaibkh_27@yahoo.com, atif-cae@nust.edu.pk
}

\begin{abstract}
In this paper, a universal steganalysis scheme for JPEG images based upon joint transform features is presented. We first analyzed two different transform domains (Discrete Cosine Transform and Discrete Wavelet Transform) separately, to extract features for steganalysis. Then a combination of these two feature sets is constructed and employed for steganalysis. A Fisher Linear Discriminant classifier is trained on features from both clean and steganographic images using all three feature sets and subsequently used for classification. Experiments performed on images embedded with two variants of F5 and Model based steganographic techniques reveal the effectiveness of proposed steganalysis approach by demonstrating improved detection for joint features.
\end{abstract}

Keywords: Steganography, Steganalysis, Information Hiding, Feature Extraction, Classification.

\section{Introduction}

The word steganography comes from the Greek words steganos and graphia, which together mean 'hidden writing'. Steganography is the art of hiding a message in plain sight. In the digital sense, it involves embedding a secret message file into an inconspicuous cover file, such as an image [1].

Steganography is an ancient subject, with its roots lying in ancient Greece and China, where it was already in use thousands of years ago. However, the modern formulation of steganography is often given in terms of the prisoners' problem [2], where Alice and Bob are two accomplices in a jail who wish to communicate in order to hatch an escape plan. However, all communication between them is examined by the warden, Wendy, who will put them in a high security prison at the slightest suspicion of covert communication. Specifically, in the general terms of a steganography model shown in Figure 1, we have Alice wishing to send a secret message $\boldsymbol{m}$ to Bob. In order to do so, she 'embeds' secret message $\boldsymbol{m}$ into a cover-object $\boldsymbol{c}$ according to a shared secret key $\boldsymbol{k}$ to obtain the stego-object $\boldsymbol{s}$. The stego-object $s$ is then sent by Alice through the public channel to Bob, $\boldsymbol{m}$ unnoticed by Wendy. Once Bob receives the stego-object $\boldsymbol{s}$, he is able to recover the secret message $\boldsymbol{m}$ using the shared secret key $\boldsymbol{k}$.

T. Wada, F. Huang, and S. Lin (Eds.): PSIVT 2009, LNCS 5414, pp. $965-975,2009$.

(C) Springer-Verlag Berlin Heidelberg 2009 


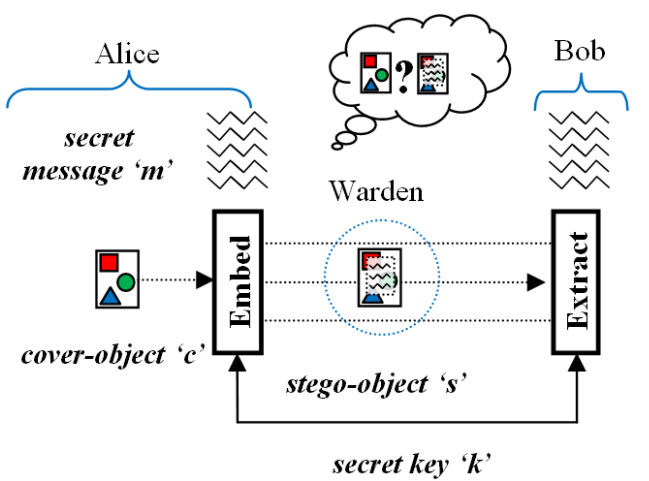

Fig. 1. A general steganography model

Steganography and cryptography are closely related data hiding methods. The purpose of cryptography is to scramble a message so that it cannot be understood, while that of steganography is to hide the message so that it cannot be seen. In general a message in cipher text might arouse suspicion on an observer while an 'invisible' message created with steganographic methods will not. Sometimes, steganography and cryptography are combined in a way that the message may be encrypted before hiding to provide additional security. Those who conceal communications through steganography are countered by those who wish to unveil such communications. The field devoted to counter steganography is known as steganalysis. The first and foremost goal of a steganalyst is to detect the presence of steganography so that the secret message may be stopped before it is received. Then the second goal is to identify the steganography tool so that the secret message may be spoofed and/or corrupted or even extracted from the stego file.

Generally, two approaches are followed for steganalysis; one is to come up with a steganalysis method specific to a particular steganographic algorithm. The other is to develop universal steganalysis techniques which are independent of the steganographic algorithm. Both approaches have their own strengths and weaknesses. A steganalysis technique specific to an embedding method would give very good results when tested only on that embedding method; but might fail on all other steganographic algorithms as in [4, 5, 6] and [7. On the other hand, a steganalysis technique which is independent of the embedding algorithm might perform less accurately overall but still shows its effectiveness against new and unseen embedding algorithms as in [8], 9], 10] and [11. Our research work is concentrated on the second approach due to its wide applicability.

In this paper, we propose a steganalysis technique by extracting features from two transform domains; the discrete wavelet transform and the discrete cosine transform. The features are investigated individually and combinatorially. The rest of the paper is organized as follows: In Section 2, we discuss the previous research work related to steganalysis. In Section 3, we present our proposed approach. Experimental results are presented in Section 4. Finally, the paper is concluded in Section 5. 


\section{Related Work}

Due to the increasing availability of new steganography tools over the internet, there has been an increasing interest in the research for new and improved steganalysis techniques which are able to detect both previously seen and unseen embedding algorithms. A good survey of benchmarking of steganography and steganalysis techniques is given by Kharrazi et al. [3].

Fridrich et al. presented a steganalysis method which can reliably detect messages hidden in JPEG images using the steganography algorithm F5, and also estimate their lengths 4. This method was further improved by Aboalsamh et al. 5 by determining the optimal value of the message length estimation parameter $\beta$. Westfeld and Pfitzmann presented visual and statistical attacks on various steganographic systems including EzStego v2.0b3, Jsteg v4, Steganos v1.5 and S-Tools v4.0, by using an embedding filter and the $\chi^{2}$ statistic [6. A steganalysis scheme specific to the embedding algorithm Outguess is proposed in 7, by making use of the assumption that the embedding of a message in a stego image will be different than embedding the same into a cover image.

Avcibas et al. proposed that the correlation between the bit planes as well as the binary texture characteristics within the bit planes will differ between a stego image and a cover image, thus facilitating steganalysis [8]. Farid suggested that embedding of a message alters the higher order statistics calculated from a multi-scale wavelet decomposition [9]. Particularly, he calculated the first four statistical moments (mean, variance, skewness and kurtosis) of the distribution of wavelet coefficients at different scales and subbands. These features (moments), calculated from both cover and stego images were then used to train a linear classifier which could distinguish them with a certain success rate. Fridrich showed that a functional obtained from marginal and joint statistics of DCT coefficients will vary between stego and cover images. In particular, a functional such as the global DCT coefficient histogram was calculated for an image and its decompressed, cropped and recompressed versions. Finally the resulting features were obtained as the $L_{1}$ norm of the difference between the two. The classifier built with features extracted from both cover and stego images could reliably detect F5, Outguess and Model based steganography techniques [10]. Avcibas et al. used various image quality metrics to compute the distance between a test image and its lowpass filtered versions. Then a classifier built using linear regression showed detection of LSB steganography and various watermarking techniques with a reasonable accuracy [1].

\section{Proposed Approach}

\subsection{Feature Extraction}

Since the dimensionality of image data is huge, it is not feasible to use the complete image data directly for steganalysis. A better option is to extract a certain amount of useful data and use it to represent the image instead of the image itself for steganalysis. This useful set of data points are called features. 
The addition of a message to a cover image does not affect the visual appearance of the image but may affect some statistics. The features required for the task of steganalysis should be able to catch these minor statistical disorders that are created during the data hiding process.

In our approach, we first extract features in the discrete wavelet transform domain, followed by the discrete cosine transform domain and finally combine both extracted features to make a joint feature set.

DWT Based Features. For extraction of features in the Discrete Wavelet Transform domain, we chose three scale decomposition as proposed by Wang and Moulin [12]. Figure 2 shows the levels and selection of subbands for this decomposition. Using 'Haar' wavelet filter we obtained nine detail subbands (Horizontal $\mathbf{H}_{i}$, Vertical $\mathbf{V}_{i}$ and Diagonal $\mathbf{D}_{i}, i=1,2,3$ ) and three approximation subbands (Lowpass $\mathbf{L}_{i}, i=1,2,3$ ). We further decomposed the first scale diagonal subband $\mathbf{D}_{1}$ to improve the performance of the features $\left[12\right.$. As $\mathbf{D}_{1}$ is the finest detail subband and each of its coefficients involves diagonal differences in a four pixel block. So, $\mathbf{H}_{1}, \mathbf{V}_{1}$ and $\mathbf{D}_{1}$ will contain more information about the difference of differences between neighboring pixels.

Various statistical measures are used in our analysis. Particularly, the first three normalized moments of the characteristic function are computed. The Kpoint discrete Characteristic Function $(\mathrm{CF})$ is defined as

$$
\Phi(k)=\sum_{m=0}^{M-1} h(m) e^{\left\{\frac{j 2 \pi m k}{K}\right\}} .
$$

where $\{h(m)\}_{m=0}^{M-1}$ is the $M$ bin histogram which is an estimate of the PDF, $p(x)$ of the wavelet coefficients distribution. The $n^{\text {th }}$ absolute moment of discrete CF is defined as

$$
M_{n}^{A}=\sum_{k=0}^{K-1} \Phi(k) \sin ^{n}\left(\frac{\pi k}{K}\right) .
$$

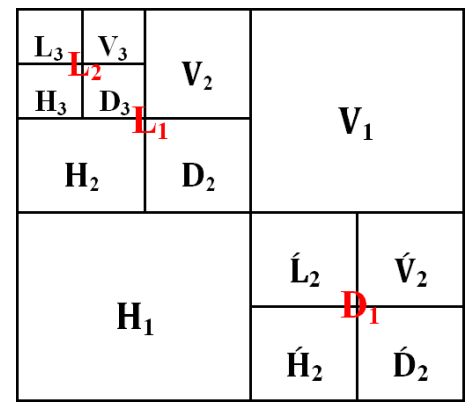

Fig. 2. A three scale wavelet decomposition 
Finally, the normalized CF moment is defined as

$$
\hat{M}_{n}^{A}=\frac{M_{n}^{A}}{M_{0}^{A}} .
$$

where $M_{0}^{A}$ is the zeroth order moment. We calculated the first three normalized $\mathrm{CF}$ moments for each of the 16 subbands, giving a 48-D feature vector.

DCT Based Features. The DCT based feature set is constructed following the approach of Fridrich [10]. A vector functional $\boldsymbol{F}$ is applied to the JPEG image $J_{1}$. This image is then decompressed to the spatial domain, cropped by 4 pixels in each direction and recompressed with the same quantization table as $J_{1}$ to obtain $J_{2}$. The vector functional $\boldsymbol{F}$ is then applied to $J_{2}$. The final feature $f$ is obtained as the $L_{1}$ norm of the difference of the functional applied to $J_{1}$ and $J_{2}$.

$$
f=\left\|\boldsymbol{F}\left(J_{1}\right)-\boldsymbol{F}\left(J_{2}\right)\right\|_{L_{1}} .
$$

The rational behind this procedure is that the recompression after cropping by 4 pixels does not see the previous JPEG compression's $8 \times 8$ block boundary and thus it is not affected by the previous quantization and hence embedding in the DCT domain. So, $J_{2}$ can be thought of as an approximation to its cover image.

We calculated the global, individual and dual histograms of the DCT coefficient array $d_{(k)}(i, j)$ as the first order functionals. The symbol $d_{(k)}(i, j)$ denotes the $(i, j)^{t h}$ quantized DCT coefficient $(i, j=1,2, \ldots, 8)$ in the $k$-th block, $(k=1,2, \ldots, B)$. The global histogram of all 64B DCT coefficients is given as, $H(m)_{m=L}^{R}$, where $L=\min _{k, i, j} d_{(k)}(i, j)$ and $R=\max _{k, i, j} d_{(k)}(i, j)$. We computed $H /\|H\|_{L_{1}}$, the normalized global histogram of DCT coefficients as the first functional.

Steganographic techniques that preserve global DCT coefficients histogram may not necessarily preserve the histogram of individual DCT modes. So, we calculated $h^{i j} /\left\|h^{i j}\right\|_{L_{1}}$, the normalized individual histograms $h(m)_{m=L}^{R}$ of 5 low frequency DCT modes, $(i, j)=(2,1),(3,1),(1,2),(2,2),(1,3)$ as the next five functionals.

The dual histogram is an $8 \times 8$ matrix which indicates the number of how many times the value ' $d$ ' occurs as the $(i, j)^{\text {th }}$ DCT coefficient over all blocks $B$ in the image. We computed $g_{i j}^{d} /\left\|g_{i j}^{d}\right\|_{L_{1}}$, the normalized dual histograms where $g_{i j}^{d}=\sum_{k=1}^{B} \delta\left(d, d_{(k)}(i, j)\right)$ for 11 values of $d=-5,-4, \ldots, 4,5$.

Inter block dependency is captured by the second order features variation and blockiness. Most steganographic techniques add entropy to the DCT coefficients which is captured by the variation $(V)$

$V=\frac{\sum_{i, j=1}^{8} \sum_{k=1}^{\left|I_{r}\right|-1}\left|d_{I_{r}(k)}(i, j)-d_{I_{r}(k+1)}(i, j)\right|+\sum_{i, j=1}^{8} \sum_{k=1}^{\left|I_{c}\right|-1}\left|d_{I_{c}(k)}(i, j)-d_{I_{c}(k+1)}(i, j)\right|}{|I r|+|I c|}$. 
where $I_{r}$ and $I_{c}$ denote the vectors of block indices while scanning the image 'by rows' and 'by columns' respectively.

Blockiness is calculated from the decompressed JPEG image and is a measure of discontinuity along the block boundaries over all DCT modes over the whole image. The $L_{1}$ and $L_{2}$ blockiness $\left(B_{\alpha}, \alpha=1,2\right)$ is defined as

$$
B_{\alpha}=\frac{\sum_{i=1}^{\lfloor(M-1) / 8\rfloor} \sum_{j=1}^{N}\left|x_{8 i, j}-x_{8 i+1, j}\right|^{\alpha}+\sum_{j=1}^{\lfloor(N-1) / 8\rfloor} \sum_{i=1}^{M}\left|x_{i, 8 j}-x_{i, 8 j+1}\right|^{\alpha}}{N\lfloor(M-1) / 8\rfloor+M\lfloor(N-1) / 8\rfloor}
$$

where $x_{i, j}$ are the grayscale intensity values of an image with dimensions $M \times N$.

The final DCT based feature vector is 20-D (Histograms: 1 global, 5 individual, 11 dual. Variation: 1. Blockiness: 2).

Joint Features. After extracting the features in the discrete cosine transform and the discrete wavelet transform domain, we finally combine the extracted feature sets into one joint feature set, giving a 68-D feature vector, (48 DWT +20 DCT).

\subsection{Classifier}

We used the two class Fisher Linear Discriminant (FLD) classifier [19]. Let $\boldsymbol{x}_{\boldsymbol{i}}, i=1, \ldots, N_{x}$ and $\boldsymbol{y}_{\boldsymbol{j}}, j=1, \ldots, N_{y}$ represent the samples from each of the two classes of the training set. The within class means are given by

$$
\boldsymbol{m}_{\boldsymbol{x}}=\frac{1}{N_{x}} \sum_{i=1}^{N x} \boldsymbol{x}_{\boldsymbol{i}}, \boldsymbol{m}_{\boldsymbol{y}}=\frac{1}{N_{y}} \sum_{j=1}^{N y} \boldsymbol{y}_{\boldsymbol{j}}
$$

The between class mean is

$$
\boldsymbol{m}=\frac{1}{N_{x}+N_{y}}\left(\sum_{i=1}^{N x} x_{i}+\sum_{j=1}^{N y} y_{j}\right)
$$

The within class scatter matrix is

$$
S_{w}=M_{x} M_{x}^{T}+M_{y} M_{y}^{T} .
$$

where $M_{x}=\boldsymbol{x}_{\boldsymbol{i}}-\boldsymbol{m}_{\boldsymbol{x}}, M_{y}=\boldsymbol{y}_{\boldsymbol{j}}-\boldsymbol{m}_{\boldsymbol{y}}$ are the matrices containing the zeromeaned $i^{\text {th }}$ and $j^{\text {th }}$ samples respectively. The between class scatter matrix is

$$
S_{b}=N_{x}\left(\boldsymbol{m}_{\boldsymbol{x}}-\boldsymbol{m}\right)\left(\boldsymbol{m}_{\boldsymbol{x}}-\boldsymbol{m}\right)^{T}+N_{y}\left(\boldsymbol{m}_{\boldsymbol{y}}-\boldsymbol{m}\right)\left(\boldsymbol{m}_{\boldsymbol{y}}-\boldsymbol{m}\right)^{T} .
$$

The maximal generalized eigenvalue eigenvector $\boldsymbol{e}$ is related to $S_{b}$ and $S_{w}$ by

$$
S_{b} e=\lambda S_{w} e
$$


By projecting the training samples $\boldsymbol{x}_{\boldsymbol{i}}$ and $\boldsymbol{y}_{\boldsymbol{j}}$ onto one dimensional linear subspace $\boldsymbol{e}\left(x_{p}=\boldsymbol{x}_{\boldsymbol{i}}^{\boldsymbol{T}} \boldsymbol{e}, y_{p}=\boldsymbol{y}_{\boldsymbol{j}}^{\boldsymbol{T}} \boldsymbol{e}\right)$, the within class scatter is minimized and the between class scatter is maximized. In any classification problem, this effect is highly desirable as it maintains the discriminability while simultaneously reduces the dimensions of data. An unknown sample $\boldsymbol{z}$ can now be tested for its class by projecting it onto the same subspace $\boldsymbol{e}\left(z_{p}=\boldsymbol{z}^{\boldsymbol{T}} \boldsymbol{e}\right)$ and its class determined on the basis of a threshold $T_{h}$.

\section{Experimental Results}

\subsection{Image Datasets}

Cover Image Dataset. For our experiments, we used 1338 grayscale images of size 512x384 obtained from the Uncompressed Colour Image Database (UCID) constructed by Schaefer and Stich [13, available at [14. These images contain a wide range of indoor/outdoor, daylight/night scenes, providing a real and challenging environment for a steganalysis problem. All images were converted to JPEG at $80 \%$ quality for our experiments.

F5 Stego Image Dataset. Our first stego image dataset is generated by the steganography software F5 [15, proposed by Andreas Westfeld. F5 steganography algorithm embeds information bits by incrementing and decrementing the values of quantized DCT coefficients from compressed JPEG images [16]. F5 also uses an operation known as 'matrix embedding' in which it minimizes the amount of changes made to the DCT coefficients necessary to embed a message of certain length. Matrix embedding has three parameters $(c, n, k)$, where $c$ is the number of changes per group of $n$ coefficients, and $k$ is the number of embedded bits. These parameter values are determined by the embedding algorithm.

F5 algorithm first compresses the input image with a user defined quality factor before embedding the message. We chose a quality factor of 80 for stego images. Messages were successfully embedded at rates of $0.05,0.10,0.20,0.3$, 0.40 and $0.60 \mathrm{bpc}$ (bits per non-zero DCT coefficients). We chose F5 because recent results in [8], 9], [12] have shown that F5 is harder to detect than other commercially available steganography algorithms.

MB Stego Image Dataset. Our second stego image dataset is generated by the Model Based steganography method [17], proposed by Phil Sallee [18]. The algorithm first breaks down the quantized DCT coefficients of a JPEG image into two parts and then replaces the perceptually insignificant component with the coded message signal. The algorithm has two types; MB1 is normal steganography and MB2 is steganography with deblocking. The deblocking algorithm adjusts the unused coefficients to reduce the blockiness of the resulting image to the original blockiness. Unlike F5, the Model Based steganography algorithm does not recompress the cover image before embedding. We embed at rates of $0.05,0.10,0.20,0.3,0.400 .60$ and $0.80 \mathrm{bpc}$. The model based steganography algorithm has also shown high resistance against steganalysis techniques in 3$],[10$. 
Table 1. The number of images in the stego image datasets given the message length. F5 with matrix embedding turned off $(1,1,1)$ and turned on $(c, n, k)$. Model based steganography without deblocking (MB1) and with deblocking (MB2). (U = unachievable rate).

\begin{tabular}{|l|c|c|c|c|}
\hline $\begin{array}{l}\text { Embedding } \\
\text { Rate (bpc) }\end{array}$ & $\begin{array}{c}\text { F5 } \\
(1,1,1)\end{array}$ & $\begin{array}{c}\text { F5 } \\
(c, n, k)\end{array}$ & MB1 & MB2 \\
\hline 0.05 & 1338 & 1338 & 1338 & 1338 \\
\hline 0.10 & 1338 & 1338 & 1338 & 1338 \\
\hline 0.20 & 1338 & 1337 & 1338 & 1334 \\
\hline 0.30 & 1337 & 1295 & 1338 & 1320 \\
\hline 0.40 & 1332 & 5 & 1338 & 1119 \\
\hline 0.60 & 5 & $\mathrm{U}$ & 1332 & 117 \\
\hline 0.80 & $\mathrm{U}$ & $\mathrm{U}$ & 60 & $\mathrm{U}$ \\
\hline
\end{tabular}

The reason for choosing the message length proportional to the number of non-zero DCT coefficients was to create a stego image database for which the steganalysis is roughly of the same level of difficulty. We further carried out embedding at different rates to observe the steganalysis performance for messages of varying length. It can be seen in Table 1 that the Model based steganography is more efficient in embedding as compared to F5; since longer messages can be accommodated in images using Model based steganography.

\subsection{Evaluation of Results}

The Fisher Linear Discriminant classifier described in Section 3.2 was utilized for our experiments. Each steganographic algorithm was analyzed separately for the evaluation of the steganalytic classifier. For a fixed relative message length, we created a database of training images comprising 669 cover and 669 stego images. Both DWT based features (DWT) and DCT based features (DCT) were extracted from the training set and were combined to form a Joint feature set (JNT), according to the procedure explained in Section 3.1. The FLD classifier was then tested on the features extracted from a different database of test images comprising 669 cover and 669 stego images. The Receiver Operating Characteristics (ROC) curves, which give the variation of the Detection Probability $\left(P_{d}\right.$, the fraction of correctly classified stego images) with the False Alarm Probability ( $P_{f}$, the fraction of stego images wrongly classified as cover image), were computed for each steganographic algorithm and embedding rate. The area under the ROC curve (AUC) was measured to determine the overall classification accuracy.

Figures 35 give the obtained ROC curves for the steganographic techniques under test for different embedding rates. Note that due to the space limitation, these figures are displayed in small size. However, readers are encouraged to take a look by using zoom to $400 \%$. We observe that the DCT based features outperform the DWT based features for all embedding rates. As could be expected, the detection of F5 without matrix embedding is better than F5 with matrix 


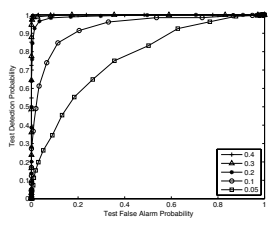

(a)

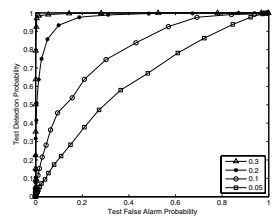

(b)

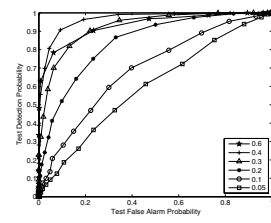

(c)

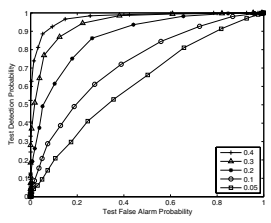

(d)

Fig. 3. ROC curves using DCT based features. (a) F5 (without matrix embedding) (b) F5 (with matrix embedding) (c) MB1 (without deblocking) (d) MB2 (with deblocking).

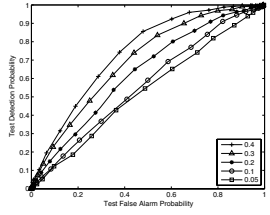

(a)

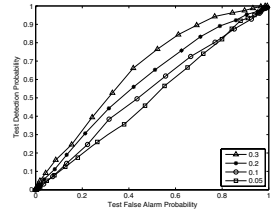

(b)

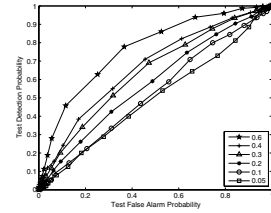

(c)

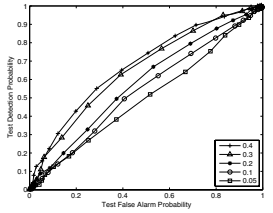

(d)

Fig. 4. ROC curves using DWT based features. (a) F5 (without matrix embedding) (b) F5 (with matrix embedding) (c) MB1 (without deblocking) (d) MB2 (with deblocking).

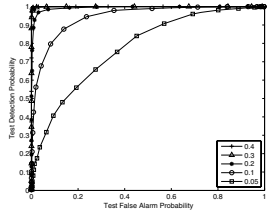

(a)

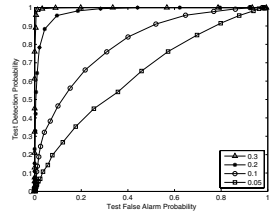

(b)

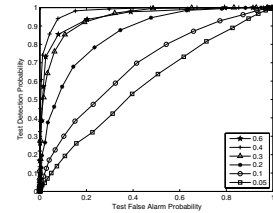

(c)

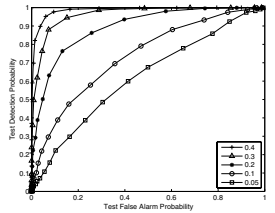

(d)

Fig. 5. ROC curves using Joint features. (a) F5 (without matrix embedding) (b) F5 (with matrix embedding) (c) MB1 (without deblocking) (d) MB2 (with deblocking).

embedding as the matrix embedding operation significantly reduces detectability at the expense of message capacity.

Table 2 summarizes the classification results. For F5 without matrix embedding, the proposed Joint transform features dominate both DCT and DWT based features for embedding rates till $0.20 \mathrm{bpc}$. For higher embedding rates the DCT based features perform better. For F5 with matrix embedding, both the proposed joint features and the DCT based features are close competitors.

For MB1 algorithm (without deblocking), the proposed joint features outperform both the DCT and DWT based features for all embedding rates. For MB2 algorithm (with deblocking), the joint features perform close to the DCT based features. It is observed that the detection of MB1 is better than MB2, as the deblocking algorithm in MB2 reduces the blockiness of the stego image to match the original image. 
Table 2. Classification results (AUC) using FLD for all embedding rates. F5 with matrix embedding turned off $(1,1,1)$ and turned on $(c, n, k)$. Model based steganography without deblocking (MB1) and with deblocking (MB2). ( $\mathrm{U}=$ unachievable rate).

\begin{tabular}{|l|c|c|c|c|c|}
\hline $\begin{array}{l}\text { Embedding } \\
\text { Rate (bpc) }\end{array}$ & $\begin{array}{c}\text { F5 } \\
(1,1,1)\end{array}$ & $\begin{array}{c}\text { F5 } \\
(c, n, k)\end{array}$ & MB1 & MB2 & \\
\hline 0.05 & 0.767 & 0.640 & 0.622 & 0.598 & DCT \\
\hline 0.05 & 0.531 & 0.491 & 0.498 & 0.492 & DWT \\
\hline 0.05 & 0.770 & 0.636 & 0.614 & 0.588 & JNT \\
\hline 0.10 & 0.932 & 0.794 & 0.732 & 0.692 & DCT \\
\hline 0.10 & 0.562 & 0.528 & 0.535 & 0.520 & DWT \\
\hline 0.10 & 0.943 & 0.796 & 0.729 & 0.686 & JNT \\
\hline 0.20 & 0.993 & 0.971 & 0.872 & 0.841 & DCT \\
\hline 0.20 & 0.624 & 0.592 & 0.581 & 0.580 & DWT \\
\hline 0.20 & 0.993 & 0.966 & 0.890 & 0.849 & JNT \\
\hline 0.30 & 0.998 & 0.998 & 0.942 & 0.924 & DCT \\
\hline 0.30 & 0.689 & 0.653 & 0.654 & 0.639 & DWT \\
\hline 0.30 & 0.993 & 0.996 & 0.954 & 0.931 & JNT \\
\hline 0.40 & 0.999 & $\mathrm{U}$ & 0.972 & 0.965 & DCT \\
\hline 0.40 & 0.735 & $\mathrm{U}$ & 0.667 & 0.666 & $\mathrm{DWT}$ \\
\hline 0.40 & 0.997 & $\mathrm{U}$ & 0.980 & 0.978 & JNT \\
\hline 0.60 & $\mathrm{U}$ & $\mathrm{U}$ & 0.984 & $\mathrm{U}$ & $\mathrm{DCT}$ \\
\hline 0.60 & $\mathrm{U}$ & $\mathrm{U}$ & 0.693 & $\mathrm{U}$ & $\mathrm{DWT}$ \\
\hline 0.60 & $\mathrm{U}$ & $\mathrm{U}$ & 0.991 & $\mathrm{U}$ & JNT \\
\hline
\end{tabular}

\section{Conclusion}

This paper presents a new DCT and DWT based joint features approach for universal steganalysis. DCT and DWT based statistical features are investigated individually, followed by research on combined features. The Fisher Linear Discriminant classifier is employed for classification. The experiments were performed on image datasets with different embedding rates for F5 and Model based steganography algorithms. Experiments revealed that for JPEG images the DCT is a better choice for extraction of features as compared to the DWT. The experiments with joint transform features reveal that the extraction of features in more than one transform domain improves the steganalysis performance.

Acknowledgments. The work on this paper was supported by the National University of Sciences and Technology, Pakistan.

\section{References}

1. Johnson, N.F., Jajodia, S.: Exploring Steganography: Seeing the Unseen. IEEE Computer 31(2), 26-34 (1998)

2. Simmons, G.J.: 'Prisoners' Problem and the Subliminal Channel. In: CRYPTO 1983-Advances in Cryptology, pp. 51-67 (1999) 
3. Kharrazi, M., Sencar, T.H., Memon, N.: Benchmarking Steganographic and Steganalysis Techniques. In: Proc. of SPIE Electronic Imaging, Security, Steganography and Watermarking of Multimedia Contents VII, San Jose, California, USA (2005)

4. Fridrich, J., Goljan, M., Hogea, D.: Steganalysis of JPEG images: Breaking the F5 Algorithm. In: Proc. 5th International Workshop on Information Hiding, Noordwijkerhout, The Netherlands, pp. 310-323 (October 2002)

5. Aboalsamh, H.A., Dokheekh, S.A., Mathkour, H.I., Assassa, G.M.: Breaking the F5 Algorithm: An Improved Approach. Egyptian Computer Science Journal 29(1), 1-9 (2007)

6. Westfeld, A., Pfitzmann, A.: Attacks on Steganographic Systems. In: Proc. 3rd Information Hiding Workshop, Dresden, Germany, pp. 61-76 (1999)

7. Fridrich, J., Goljan, M., Hogea, D.: Attacking the OutGuess. In: Proc. ACM Workshop on Multimedia and Security 2002. ACM Press, Juan-les-Pins (December 2002)

8. Avcibas, I., Memon, N., Sankur, B.: Image Steganalysis with Binary Similarity Measures. In: Proc. of the IEEE International Conference on Image Processing, Rochester, New York (September 2002)

9. Farid, H.: Detecting Hidden Messages Using Higher-order Statistical Models. In: Proc. of the IEEE International Conference on Image Processing, vol. 2, pp. 905908 (2002)

10. Fridrich, J.: Feature-Based Steganalysis for JPEG Images and its Implications for Future Design of Steganographic Schemes. In: Moskowitz, I.S. (ed.) Information Hiding 2004. LNCS, vol. 2137, pp. 67-81. Springer, Heidelberg (2005)

11. Avcibas, I., Memon, N., Sankur, B.: Steganalysis Using Image Quality Metrics. IEEE Transactions on Image Processing 12(2), 221-229 (2003)

12. Wang, Y., Moulin, P.: Optimized Feature Extraction for Learning-Based Image Steganalysis. IEEE Transactions on Information Forensics and Security 2(1) (2007)

13. Schaefer, G., Stich, M.: UCID - An Uncompressed Colour Image Database. In: Proc. SPIE, Storage and Retrieval Methods and Applications for Multimedia, San Jose, USA, pp. 472-480 (2004)

14. UCID - Uncompressed Colour Image Database (visited on 02/08/08), http://vision.cs.aston.ac.uk/datasets/UCID/ucid.html

15. Steganography Software F5 (visited on 02/08/08), http://wwwrn.inf.tu-dresden.de/ westfeld/f5.html

16. Westfeld, A.: F5 - A Steganographic Algorithm: High capacity despite better steganalysis. In: Moskowitz, I.S. (ed.) 4th International Workshop Information Hiding. LNCS, pp. 289-302. Springer, Heidelberg (April 2001)

17. Model Based JPEG Steganography Demo (visited on 02/08/08), http://www.philsallee.com/mbsteg/index.html

18. Sallee, P.: Model Based Steganography. In: International Workshop on Digital Watermarking, Seoul, Korea, pp. 174-188 (October 2003)

19. Duda, R.O., Hart, P.E., Stork, D.G.: Pattern Classification, 2nd edn. John Wiley \& Sons, New York (2001) 